\title{
Enfrentamento do pai frente à malformação congênita do filho antes e depois do nascimento
}

\section{Father coping face to the congenital malformation of son before and after the birth}

\section{Enfrentamiento del padre delante de la malformación congénita del hijo antes y después del nacimiento}

\author{
Elisabeth Hellen Pereira da Silva* \\ Universidade Federal do Rio de Janeiro - UFRJ, Rio de Janeiro, Rio de Janeiro, Brasil
}

Emmanuela da Rocha Cruz Girão**

Universidade Federal do Rio de Janeiro - UFRJ, Rio de Janeiro, Rio de Janeiro, Brasil

\section{Ana Cristina Barros da Cunha***}

Universidade Federal do Rio de Janeiro - UFRJ, Rio de Janeiro, Rio de Janeiro, Brasil

\begin{abstract}
RESUMO
Todo casal idealiza um filho saudável, o que não ocorre quando existe uma malformação congênita. Poucos estudos consideram as expectativas e enfrentamento paternos diante da malformação de seu filho, objetivo deste estudo. Investigaram-se possíveis diferenças em indicadores de depressão e modos de enfrentamento em 12 pais divididos em dois grupos: grávidos e com filhos entre 3 e 5 anos. O diagnóstico da malformação ocorreu durante a gravidez de suas mulheres, atendidas em uma maternidade pública do Rio de Janeiro. Foram utilizados o Questionário "Momento da Notícia", o Inventário de Depressão (BDI) das Escalas Beck e a Escala Modos de Enfrentamento de Problemas (EMEP). No total dos pais, constatou-se que $66,7 \%$ recebeu o diagnóstico por médicos, considerando a comunicação do diagnóstico como positiva. Observou-se que $75 \%$ dos pais reagiram negativamente, ainda que as repercussões da malformação para o casal tenham sido avaliadas como positiva por $83,33 \%$ do total de pais. Sentimentos negativos, como tristeza, foram relatados por todos os pais, ainda que $91,2 \%$ dos pais de ambos os grupos apresentaram sinais mínimos de depressão. O enfrentamento mais prevalente pela EMEP para ambos os grupos foi o "Focalizado no Problema", seguido de "Busca de práticas religiosas". Embora sem diferenças entre os grupos, conclui-se que a malformação do filho representa impacto emocional no enfrentamento paterno.
\end{abstract}

Palavras-chave: pai, malformação congênita, enfrentamento, depressão.

\section{ABSTRACT}

Every couple idealizes a healthy son, which not happened when the congenital malformation exists. Few studies consider the paternal 
expectations and the father's coping face to the son malformation, the aim of this study. We investigated differences on the indicators of depression and coping in 12 parents, divided into two groups: pregnant fathers and fathers with children from 3 to 5 years old. The diagnosis of the child's malformation occurred during the pregnancy of their wives attended in a public maternity hospital in the Rio de Janeiro city. The Questionnaire "Momento da Noticia" (Moment of diagnosis news), the Depression Inventory (BDI) of the Beck Scale, and the EMEP Scale (Ways of Coping with Problems Scale) were used. For all parents, it was found that $66.7 \%$ received the diagnosis by doctors, and considered the communication of diagnosis was positive. It was observed that $75 \%$ of parents reacted negatively, even if the repercussions of the malformation to the couple have been considered as positive by $83,33 \%$ of parents. Negative feelings, such as sadness, were reported by all parents, even though $91.2 \%$ of the parents of both groups showed minimal signs of depression. The most prevalent way of coping by EMEP for both groups was the "Focused on the Problem", followed by "Search of religious practices". Although we did not find differences between the groups, we concluded that the malformation of the child has emotional impact on the father coping.

Keywords: father, congenital malformation, coping, depression.

\section{RESUMEN}

Cada pareja concibe un hijo sano, lo que no ocurre cuando hay una malformación congénita. Pocos estudios consideran las expectativas y afrontamiento de los padres antes de la malformación de su hijo, el objetivo de este estudio. Se investigaron las diferencias en los indicadores de depresión y afrontamiento en 12 padres, divididos en dos grupos: padres embarazados y padres con niños de 3 a 5 años de edad. El diagnóstico de la malformación del niño ocurrió durante el embarazo de sus esposas atendidas en una maternidad pública de la ciudad de Río de Janeiro. El Cuestionario "Momento da Noticia", el Inventario de Depresión (BDI) de la Escala de Beck Escala Modos de Afrontamiento (EMEP), fueron utilizados. En todos los padres, para $66,7 \%$ el diagnóstico de la malformación fue dada por los médicos, dando se cuenta de la comunicación del diagnóstico como positivo. Se observó que el $75 \%$ de los padres reaccionó negativamente, aunque las repercusiones de la malformación en la pareja han sido evaluadas como positivos por $83,33 \%$ de los padres. Los sentimientos negativos, como la tristeza, fueron reportados por todos los padres, a pesar de que el $91,2 \%$ de los padres de ambos grupos mostraron signos mínimos de depresión. El afrontamiento más frecuente por EMEP para ambos grupos fue el "Centrado en el problema", seguido de "Búsqueda de las prácticas religiosas". Aunque no se encontraron diferencias entre los grupos, se concluye que la malformación del niño tiene impacto emocional en el afrontamiento de los padres.

Palabras-claves: padre, malformación congénita, afrontamiento, la depresión.

\section{Introdução}

A gestação é um período complexo que resulta em experiências psicológicas e fisiológicas peculiares a esta condição, com modificações nas relações sociais, desde a descoberta da gravidez até 
o nascimento do bebê (Vasconcelos \& Petean, 2009). Dessa forma, conforme o bebê se desenvolve, as percepções maternas e paternas surgem e o casal cria expectativas e idealizações sobre um bebê que seja saudável (Machado, 2012). É durante a gravidez que essas fantasias do bebê saudável podem ou não se confirmar, dependendo dos resultados de exames realizados ainda durante o pré-natal. Segundo Machado (2012): “desde os importantes avanços tecnológicos da medicina fetal, tais como a ultrassonografia, tem-se tornado cada vez mais eficiente o rastreamento e o diagnóstico da malformação fetal intra-útero ${ }^{1 " \prime}$ (p. 88).

No caso de um diagnóstico de malformação, o que foi imaginado e desejado para o filho passa a se diferenciar bastante do real e, por conta disso, os cuidadores deixam de realizar projeções futuras relacionados a si mesmos e a prole (Gomes \& Piccinini, 2010). O nascimento de um bebê com malformação congênita produz, portanto, a descontinuidade no processo de idealização do nascimento de um bebê saudável, uma vez que os sonhos sobre a prole desmoronam e, consequentemente, surgem sentimentos negativos (Roecker, Mai, Baggio, Mazzola, \& Marcon, 2012) para a mãe, o pai e a família.

Dentre os cuidadores, destacam-se os pais, personagem principal do nosso estudo. Historicamente o papel do pai na instituição familiar vem se reformulando, com maior participação no desenvolvimento dos filhos desde a década de 1970. O homem passou a ser convocado para tomar parte na responsabilidade de cuidar dos filhos (Cia, Williams, \& Aiello, 2005), contestando-se crenças de que ele estabelece uma ligação afetiva com o filho menos intensa do que a mãe, por ser reconhecido como sinônimo de força e de distanciamento afetivo (Barros, Menandro, \& Trindade, 2006). De acordo com Barros, Menandro, \& Trindade (2006), é importante reconhecer os sentimentos paternos questionando tais crenças, além de reconsiderar o papel do pai nos modelos de cuidados familiares, antes exclusivamente direcionados para mãe.

Apesar de tradicionalmente a depressão materna ser tema principal da maioria das investigações, que reafirmam a ideia de que a notícia do comprometimento de um filho gera estresse, sobrecarga emocional, ansiedade e depressão (Perosa, Canavez, Silveira, Padovani, \& Peraçoli, 2009), pais de bebês diagnosticados com malformação congênita também podem sofrer esse impacto, com todas as implicações de uma gravidez de risco (Bornholdt, Wagner , \& Staudt, 2007). Estudos sobre o impacto da malformação congênita de um filho indicam que a descoberta deste tipo de diagnóstico envolve momentos distintos com experiências emocionais singulares (Roecker et al., 2012; Benute et al, 2012). De acordo com Roecker et al. (2012) em cada um destes momentos se deflagra uma crise pautada em mecanismos de negação, os quais, paulatinamente, são 
substituídos por estratégias mais ou menos adaptativas e resolutivas para lidar com a situação. Dessa forma, a gravidez com malformação repercute em um estado emocional inicial de maior insegurança, medos e angústias, que culminam em estresse significativo para todos os envolvidos (Benute et al, 2012).

Nesta perspectiva, adotamos como objeto de estudo o enfrentamento paterno da malformação de um filho, dando, assim, importância à vivência psicológica de pais que recebem a notícia de malformação congênita, com ênfase na investigação de indicadores de depressão paterna no pré e pós-parto. Depressão pré e pós-natal é transtorno grave que, além de gerar comprometimentos de diferentes níveis no ciclo gravídico-puerperal, pode acometer tanto mãe quanto pai. Revisão da literatura PSI indica que a depressão pós-natal é universal e ocorre em diferentes culturas e grupos socioeconômicos, com efeitos nas interações precoces e práticas parentais, além de comprometimento do exercício da parentalidade na rotina de cuidados ao bebê, como amamentação, sono, vacinas etc. (Field, 2010).

No entanto, a depressão paterna é tema pouco conhecido, já que o interesse dos pesquisadores pela relação pai-bebê é recente e, de modo geral, centram-se na valorização desta relação (pai-bebê) após o nascimento (Piccinini, Silva, Gonçalves, Lopes, \& Tudge, 2004), sem que se tenha registros de programas de rastreio de depressão no período pós-natal em pais (Edmondson, Psychogiou, Vlachos, Netsi, \& Ramchandani, 2010). Ainda que pouco notificada devido às dificuldades do público masculino acessar os serviços de saúde mental (Edmondson et al., 2010), a depressão pós-natal em pais tem sido ainda relacionada a efeitos adversos sobre o desenvolvimento da criança (Fisher, Kopelman , \& O'Hara, 2012; Massoudi, Hwang , \& Wickberg, 2013) e sintomas psicopatológicos com efeitos emocionais e comportamentais negativos em crianças (Edmondson et al., 2010). Por conseguinte, a depressão pós-natal paterna tem sido associada com a depressão pós-parto materna e costuma ter seu ápice entre o 3o e 6으 mês após o parto (laconelli, 2011). Sintomas depressivos parecem ocorrer devido ao estresse relacionado ao "medo do homem de falhar nas tarefas de provedor, apoiador emocional e parceiro romântico" (Falceto, Fernandes, \& Kerber, 2012, p. 294), que podem se agravar diante de um diagnóstico de malformação do filho. Cabe ainda ressaltar que, por questões culturais e de gênero, os homens têm dificuldade de reconhecer a depressão como uma doença e muitas vezes não têm seu sofrimento devidamente reconhecido pela família e pela equipe hospitalar (I aconelli, 2011).

No que diz respeito à depressão pré-natal paterna, estudo de Locock e Alexander (2006) sugere que as mulheres não reconhecem o impacto das complicações da gravidez sobre os homens, já que eles escondem 0 que sentem. Muitas vezes o próprio homem tem 
dificuldade de reconhecer seu sofrimento e seus sinais de depressão frente à notícia de malformação do filho. Além disso, o pai continua sendo cobrado pelos diversos contextos em que está inserido, que lhe exigem a manutenção de seu desempenho masculino como se não existisse o reconhecimento do seu sofrimento e os possíveis sinais de um quadro psicopatológico. Logo, se faz necessário estar atento às alterações psicológicas que homens também podem apresentar após a notícia de malformação de um filho a fim de cuidar não apenas da gestante, mas também do seu companheiro, durante e após a gestação.

Outro ponto que nos interessa diz respeito às estratégias de coping ou enfrentamento ${ }^{2}$ utilizadas pelos pais para lidar com o impacto da notícia do diagnóstico de malformação congênita, assim como os sentimentos decorrentes. Devido seu impacto pessoal e familiar, a malformação é considerada um evento estressor, sendo importante investigar as estratégias de enfrentamento, ou seja, os comportamentos, manifestos ou não, adotadas pelos pais para minimizar o choque pela perda do filho idealizado. Parte-se do modelo de compreensão do coping com um processo dinâmico de enfrentamento de situações de estresse que envolve estratégias que são aprendidas, usadas e adaptadas com base na percepção e na interpretação do evento estressor (Carver \& Connor-Smith, 2010). Dessa forma, esse enfrentamento, que pode se focar no problema, na emoção ou reunir ambos, pode incluir diferentes tipos de estratégias, como a religiosidade, por exemplo, adotadas simultaneamente no enfrentamento de uma situação específica para auxiliar na elaboração psíquica das repercussões de uma condição cronicamente estressora, como a malformação congênita, por exemplo.

Em nossa pesquisa constatamos, através das bases virtuais do Scielo.br, BVSalud.org e Portal de Periódicos Capes, grande escassez de trabalhos sobre o enfrentamento paterno, diante da malformação congênita do filho. Assim sendo, consideramos socialmente relevante o tema aqui abordado, uma vez que o papel paterno no desenvolvimento da criança, apesar de importante, tem sido pouco discutido, principalmente no que se refere à função do pai como protetor do desenvolvimento.

A revisão da literatura PSI sobre paternidade reafirma a relevância da figura paterna para o desenvolvimento infantil, quando ao pai é atribuído funções diversas relacionadas às diferentes etapas desenvolvimentais, desde a gestação (Vieira et al., 2014). Tais funções englobam o cuidar associado ao educar, com base em um envolvimento paterno mais afetivo e participativo (Bossardi \& Vieira, 2010). No entanto, ainda que prevaleça a ideia de que o pai tenha um papel ativo na educação e cuidado dos filhos, isto vem ocorrendo de forma compartilhada, mas não igualitária com a mãe, quando o pai se mantém como coadjuvante dela no exercício dos cuidados 
parentais (Vieira et al., 2014). Possivelmente, isto acaba por contribuir e reforçar o conceito, historicamente construído, da paternidade pautada na representação do homem exclusivamente como provedor financeiro do lar.

Diante do exposto, objetivamos estudar o enfrentamento paterno da malformação congênita de um filho com base na investigação de possíveis diferenças em indicadores de depressão, e também no modo de enfrentamento (coping) entre pais grávidos e pais com filhos entre 3 e 5 anos de idade, ambos tendo recebido a notícia de malformação congênita ainda na gestação.

\section{Método}

\subsection{Participantes}

Com base em um delineamento descritivo participaram da pesquisa 12 pais com filhos diagnosticados com malformação congênita, cujas mulheres foram atendidas no período de 2009 a 2014 em uma maternidade pública com caráter de ensino na cidade do Rio de Janeiro. Trata-se de uma amostra de conveniência constituída por usuários de um ambulatório de follow-up de neurocirurgia infantil, cujos filhos tinham sido diagnosticados com malformação congênita durante a gravidez nos períodos de 2009 a 2011, passaram por cirurgia corretiva na própria instituição logo após o nascimento e eram acompanhados no referido ambulatório até a idade de 5 anos.

Para responder ao objetivo do estudo a amostra foi dividida em dois grupos. O primeiro grupo, chamado Grupo Retrospectivo (GR), foi composto por 6 pais de crianças com idades entre 03 e 05 anos, no momento da pesquisa. Os pais desse grupo tinham como média de idade 35 anos e todos se declararam casados ou em união estável, com média de 5,6 anos de relacionamento conjugal, exercendo profissões como pedreiro, garagista, chefe de segurança, entre outras.

O segundo grupo, chamado Grupo Atual (GA), foi composto por 6 companheiros de gestantes sob acompanhamento no Setor de Medicina Fetal com diagnostico de malformação congênita confirmado, em geral, no 2 o trimestre de gestação. A média de idade destes pais foi de 34 anos, três se declararam casados, um participante declarou união estável e dois solteiros, sendo a média do tempo de união de 8,5 anos, com relato de profissões como técnico em telecomunicações, gerente gráfico, entre outras.

\subsection{I nstrumentos}


Para avaliação do enfrentamento diante da malformação congênita do filho foram utilizados o Questionário "Momento da Notícia", a Escala Modos de Enfrentamento de Problemas (EMEP) e o Inventário de Depressão (BDI) das Escalas Beck, que serão brevemente descritos a seguir.

O Questionário "Momento da notícia" é um instrumento adaptado de Ramos, Hoffmann, \& Regen (1985), composto por perguntas abertas e fechadas que tem por objetivo avaliar quais foram as primeiras reações do pai após a notícia do diagnóstico do bebê, como a notícia foi comunicada e recebida por ele, além das possíveis estratégias de enfrentamento utilizadas por eles e como se sentem atualmente diante da condição do filho. Diferente dos pais do Grupo Atual, para os pais do Grupo Retrospectivo, esse instrumento foi aplicado solicitando que eles formulassem suas respostas recordando 0 momento em que a notícia foi dada pela primeira vez.

Outro instrumento utilizado foi a Escala Modos de Enfrentamento de Problemas (EMEP), versão adaptada de Seidl, Tróccoli e Zannon (2001) para avaliar as estratégias de enfrentamento frente a estressores específicos. A EMEP é uma escala com 45 itens que disponibilizam 5 alternativas de resposta numa escala tipo Likert com pontos dispostos da seguinte maneira: 1- Eu nunca faço isso; 2- Eu faço isso um pouco; 3- Eu faço isso às vezes; 4- Eu faço isso muito; e 5- Eu faço isso sempre. Dentre estes 45 itens, distribuídos de modo não-ordenado na escala, 18 correspondem ao Fator 1 Enfrentamento focalizado no problema; 15 itens correspondem ao Fator 2 - Enfrentamento focalizado na emoção; 7 itens correspondem ao Fator 3 - Busca de prática religiosa/pensamento fantasioso; e o Fator 4 - Busca de suporte social possui 5 itens. Na correção da EMEP os escores são obtidos pela média aritmética e, quanto mais altos estas médias, maior é a frequência de utilização da estratégia de enfrentamento.

Para avaliar indicadores de depressão nos pais foi utilizado o Inventário de Depressão (BDI), das Escalas Beck, que se propõe mensurar a intensidade da depressão. O BDI é um instrumento validado e padronizado para população brasileira por Cunha (2001) e contém 21 grupos de afirmações, apresentando quatro alternativas de resposta, que somadas resultam em um escore que classifica a depressão em quatro graus crescentes de intensidade: 1) nível 0 ou mínimo: com escores entre 0 a 10; 2) nível 1 ou leve: com escores entre 11 a 19; 3) nível 2 ou moderado: com escores entre 20 a 30; e 4) nível 3 ou grave: com escores entre 31 a 63 . O BDI é um instrumento respondido por meio de auto-relato, assim como os outros instrumentos utilizados nesta pesquisa.

\subsection{Procedimento de coleta de dados}


No Grupo Retrospectivo (GR), os pais foram convidados a participar da pesquisa quando seus filhos eram acompanhados no ambulatório de follow-up de neurocirurgia infantil. Enquanto que o contato com os pais do Grupo Atual (GA) foi feito durante a consulta de acompanhamento pré-natal no Setor de Medicina Fetal. Os participantes deste estudo foram abordados pessoalmente e a aplicação dos instrumentos foi realizada individualmente, após assinatura do Termo de Consentimento Livre e Esclarecido (TCLE), aprovado pelo Comitê de ética da instituição (CAEE n. 0033.0.361.361-9 de 28 de junho de 2010).

\subsection{Análise dos dados}

Todos os dados de avaliação de coping e depressão foram processados de acordo com as instruções dos instrumentos. Para análise do tipo de enfrentamento mais frequente nos grupos, GR e GA, pela EMEP adotou-se ainda o cálculo de medianas para cada fator por grupo, comparando-se tais medianas a fim de obter uma medida de tendência central da frequência de cada tipo de enfrentamento pelos grupos. Para análise dos dados do Questionário "Momento da Notícia", foi adotada análise quantitativa e qualitativa das respostas dos participantes registradas por escrito no próprio questionário. Os dados do Questionário foram processados e analisados utilizando-se a metodologia de análise de conteúdo de Bardin, quando se extraiu dos registros as seguintes categorias de análise de conteúdo e suas respectivas frequências de ocorrência: a) "Comunicação da notícia" (avaliação positiva, negativa); b) "Como se sentiu" (sentimentos negativos; positivos; ambivalentes); c) "Reação à notícia" (atitude positiva; negativa; ambivalente); d) "Repercussões na relação do casal" (avaliação negativa; positiva); e) "Busca de suporte social" ( sim; não); e f) "Como se sente atualmente" (sentimentos negativos; positivos; ambivalentes). E, ainda, baseado nos registros de resposta às perguntas abertas do Questionário, foram feitas análises do discurso dos participantes. Com base na proposta de processamento dos dados apresentada, foram feitas análises descritivas comparativas entre os dois grupos de pais, GA e GR, para as categorias de análise do relato verbal pelo Questionário Momento da Notícia, os níveis de depressão pelo BDI e as estratégias de enfrentamento pela EMEP.

\section{Resultados}

Os dados do Questionário "Momento da Notícia" mostraram que, em relação à categoria "Comunicação da notícia", 66,7 \% dos pais foram comunicados sobre o diagnóstico de malformação pela equipe médica 
e avaliaram positivamente a experiência. Apenas 33,3\% dos pais dos dois grupos, GR e GA, referiram terem sido informados pela companheira. No GR a postura atenciosa dos médicos foi destacada pelos pais, como pode ser observado nos relatos a seguir: a) "Não estava presente na hora, mas sei que a notícia foi dada de forma cuidadosa" (Pedro ${ }^{3}$ - Grupo Retrospectivo). Contudo, um pai do GR, Simão, percebeu como negativa a postura da médica pediatra que "Deu a notícia com muito ímpeto, condenou o filho, foi muito ríspida, disse que o filho só iria andar de cadeira de rodas", considerando, assim, que a notícia "Tinha que ter sido dada não tão direta, deveriam ter preparado mais o psicológico para a notícia".

Em relação à categoria "Como se sentiu" ao receber a notícia, 100\% dos pais de ambos os grupos (GR e GA) não relataram sentimentos positivos sobre o diagnóstico. Elias, um pai do GR, contou que se sentiu triste no momento da notícia e Ciro, um dos pais do GA, relatou que se sentiu "Triste, ansioso e com esperança que 0 problema desapareceria".

No que se refere à categoria "Reação à notícia", verificou-se que, nos dois grupos, $75 \%$ dos participantes reagiu de forma negativa, com exemplos de discursos como "Me senti sem chão, não esperava por isso" (Ananias, do GR) e "Muito triste" (Jonas, do GA). Apenas um pai reagiu de forma ambivalente referindo que "Ficou triste, mas esperançoso" e nenhum pai demonstrou ter atitude positiva frente ao diagnóstico da malformação. Dois pais não relataram como foram suas reações frente a notícia, um de cada grupo.

Sobre a categoria "Repercussões na relação do casal", ou seja, o impacto do diagnóstico no relacionamento conjugal, não houve diferença entre os grupos, $83,33 \%$ dos pais do GR e do GA relataram que ficaram mais unidos e próximos de suas companheiras/mães e que a relação do casal melhorou, como ilustram os relatos a seguir: a) "Ficamos mais unidos. O problema do bebê melhorou nossa relação. Passei a conhecer uma nova e grande mulher guerreira" (André - Grupo Retrospectivo); e b) "Passei a valorizar mais minha mulher (mãe do bebê) por todo o sofrimento que ela estava passando. E depois de todos esses problemas passaram a se unir mais, a se compreender e a se respeitarem mais" (Eliseu - Grupo Atual).

Em relação à categoria "Como se sente atualmente", observou-se que os dois grupos não apresentaram grandes diferenças em relação aos sentimentos relacionados ao momento do diagnóstico de malformação fetal. Entretanto, todos os pais do GR, quando perguntados sobre como se sentem atualmente referiram sentimentos positivos, como "esperançoso" e "tranquilo", quando comparados aos pais do GA, no qual $33,33 \%$ dos pais relataram sentimentos negativos, representados por discursos como: "preocupado" (Rafael) e "Incapaz de fazer qualquer coisa para ajudar 
o bebê e triste" (Felipe).

Ainda foi perguntado aos pais se sentiram necessidade de conversar com alguém após receber a notícia da malformação do filho, quando se verificou que três pais $(24,9 \%)$ relataram tal necessidade, sem de fato ter procurado esse suporte. Um pai referiu, por exemplo, ter sentido vontade sem ter falado com ninguém, 41,6\% ( $N=5)$ dos pais relataram ter conversado com pessoas, como amigos $\mathrm{e}$ familiares. Rúben do GR, por exemplo, relatou que recebeu "ajuda do pastor com conversas e orientações"; além disso, 33,3\% ( $N=4)$ disse que não sentiram necessidade de conversar.

Os resultados obtidos através da Escala Modos de Enfrentamento de Problemas (EMEP) estão apresentados na figura abaixo em termos das medianas de cada grupo para os fatores da escala. De modo geral, os resultados indicaram que os pais de ambos os grupos apresentaram o fator "Focalizado no Problema" como modo de enfrentamento mais prevalente para lidar com a situação do diagnóstico do filho. Como pode ser observado na Figura 1, no GR a mediana foi de 4,33 , pouco maior do que a do GA que foi 3,85 .

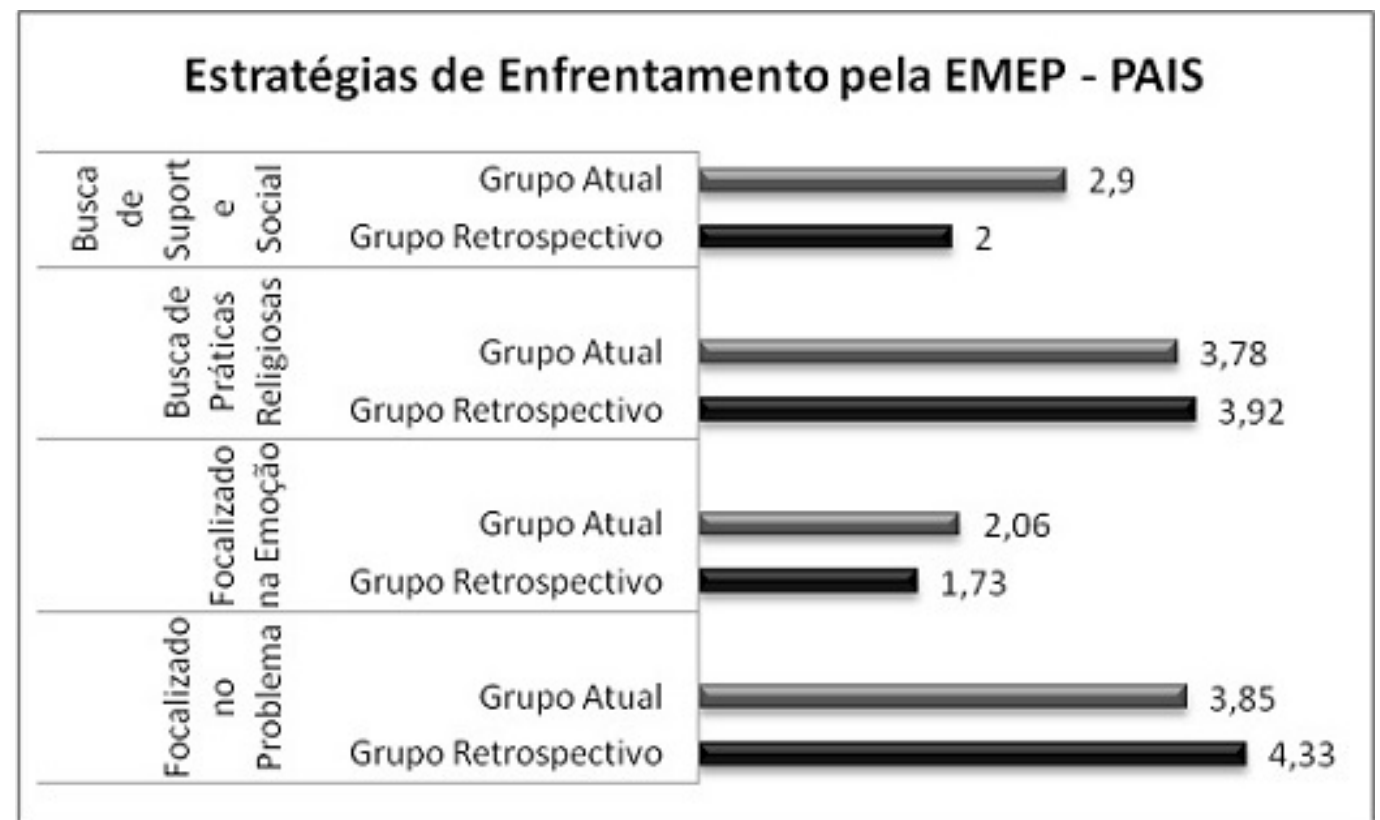

Figura 1 - Medianas das estratégias de enfrentamento dos pais pela EMEP

Também não houve grande diferença na mediana dos outros fatores da EMEP em ambos os grupos. Podemos destacar ainda o fator "Busca de prática religiosa/pensamento fantasioso", que foi o segundo mais frequente entre os pais. Neste fator, as medianas dos Grupos Retrospectivo e Atual foram, respectivamente, 3,92 e 3,78. Este fator representa a busca de comportamentos religiosos para ajudar no enfrentamento do problema, mas também o uso de pensamentos fantasiosos como forma de enfrentar a situação, com itens como "Eu sonho ou imagino um tempo melhor do que aquele 
em que estou" (item 26) e "Eu desejaria poder mudar o que aconteceu comigo" (item 41). No entanto, cabe ressaltar que, todos os pais declararam religião, católicos $(n=7)$ ou evangélicos $(n=5)$, sendo que $66,6 \%(\mathrm{~N}=8$ ) deles eram praticantes.

Analisando os resultados obtidos com o Inventário de Depressão de Beck (BDI), 91,2\% ( $N=11)$ dos pais não apresentaram indicadores de depressão consideráveis, sendo seus escores classificados no nível mínimo para ambos os grupos. Apenas um pai do GR apresentou nível moderado de depressão.

\section{Discussão}

De modo geral, a literatura PSI aborda o tema da paternidade, no contexto de malformação congênita, de maneira restrita, uma vez que as discussões costumam centrar-se na relação pai-bebê após o nascimento da criança (Piccinini et. al., 2004). Obviamente é indiscutível a importância do pai como mecanismo de proteção ao desenvolvimento infantil (Bossard \& Vieira, 2010; Cia, Williams, \& Aiello, 2005; Vieira et al., 2014) e ele deve ser alvo de atenção, tanto na sua saúde física como mental, ideia que também será defendida no nosso estudo por colaborar para a discussão proposta.

Locock e Alexander (2006) afirmam que, em geral, as mulheres não reconhecem no homem qualquer abalo que possa aparecer em decorrência de complicações na gravidez, ainda que tal afirmativa deva ser questionada à luz de autores que reafirmam o crescente envolvimento afetivo do pais com seus filhos desde a gravidez (Bossard \& Vieira, 2010). No entanto, tal percepção pela mãe pode ser justificada pelo fato de que os pais também ainda são vistos como provedores de estabilidade financeira e emocional de sua família e fonte de apoio para a mulher (Falceto, Fernandes , \& Kerber, 2012). Contudo, Bornholdt et. al. (2007) demonstraram que os homens também são atravessados pelas questões que surgem durante a gravidez, isto é, também são dignos de atenção após receberem um diagnóstico de malformação.

Através da análise do "Questionário Momento da Notícia" pode-se observar que mais da metade dos pais foram informados sobre o diagnóstico pela equipe médica e ressaltaram como importante o cuidado que observaram quando foram comunicados. Ha, Anat e Longnecker (2010) destacam a importância da comunicação entre médico e paciente para que haja compreensão da notícia transmitida. Para esses autores, essa comunicação deve ser feita de modo eficaz possibilitando a regulação das emoções pelo paciente, neste caso, os pais.

Quase 90\% dos participantes referiram repercussões positivas na relação do casal, no sentido de uma maior aproximação conjugal 
após a notícia de malformação. Inclusive, pais que relataram isso também referiram ter oferecido suporte emocional as suas companheiras/mães desde o momento da descoberta da situação do bebê. Falceto et. al (2012) consideram que faz parte da tradição da cultura brasileira o pai, além de ser o provedor, oferecer apoio a sua companheira nos cuidados com o bebê, responsabilidade primeira da mãe.

Contudo, acreditamos importante considerar que, ainda que o pai assuma essa função de suporte financeiro e instrumental no cuidado do filho em resposta ao que é culturalmente esperado, ele também deve ser considerado alvo de atenção nesses casos. As suas necessidades como indivíduo e pai precisam ser reconhecidas e atendidas (Locock \& Alexander, 2006), ainda mais quando se considera que eles não têm seu sofrimento devidamente legitimado pela família e pela equipe hospitalar (Iaconelli, 2011).

Compreendemos, ainda, que o impacto da malformação sobre o pai pode ter sido minimizado com o tempo, ou mesmo que o relato rememorado daqueles homens pode apresentar um viés na captura do impacto da notícia da malformação passado 3 a 5 anos do diagnóstico inicial. No entanto, reafirmamos a importância do pai ser cuidado pela equipe e apoiado nas suas demandas psíquicas. Relatos de pais das crianças maiores (Grupo Retrospectivo) confirmam essa hipótese, ou seja, de que passado o momento do diagnóstico e a presença das constantes demandas como cuidador podem contribuir para a elaboração do impacto inicial da notícia da malformação, antes vista como algo negativo, "muito triste" (André) e motivo, inclusive, de "choro" (Pedro), e depois encarada de forma mais positiva: "Muito seguro, com esperança" (Simão).

Assim, consideramos que o significado do diagnóstico pode ser reelaborado e a dinâmica psíquica reajustada diante das demandas como cuidador de uma criança com deficiência. Todavia, isto não diminui a importância de se atender às necessidades do homem, como pode ser observado na fala de Ananias, também pai do Grupo Retrospectivo: "Fiquei muito preocupado com minha esposa, os homens são fortes, mas às vezes fraquejam, principalmente quando vê a mãe de seu filho sofrendo". O discurso de Ananias confirma que, diante da notícia da malformação de um filho, os homens são passíveis de sofrer tanto quanto as mulheres e que, portanto, 0 cuidado e atenção ao pai deve ser uma questão sobre a qual a equipe de saúde se foque, até porque o processo de elaboração pode ser otimizado quando é oferecido também ao pai atenção integral à sua saúde física e mental.

Entretanto, nem sempre a equipe de saúde reconhece o sofrimento psicológico do pai (Iaconelli, 2011) e centra sua atenção sobre a mulher. Estudo realizado com pais suecos (Jungmarker, Lindgren, \& Hildingsson, 2010) mostrou que eles se sentem excluídos pelos 
profissionais de saúde durante a gravidez, uma vez que, ao contrário das mães que recebem apoio da equipe médica e suporte social da sua própria família, da família do cônjuge e de amigos, os pais são menos favorecidos em relação ao suporte social e emocional desses grupos de apoio. Como já dito, tal quadro é reflexo de uma cultura que tradicionalmente considera o pai como alguém que não tem necessidade de ser apoiado, justamente por ter um papel de apoiador e provedor pré-definido.

Com relação aos resultados da EMEP, a principal estratégia identificada no enfrentamento dos pais foi a "Focalizado no problema", que apareceu também como segunda mais prevalente para as mães. De acordo com Seidl et. al. (2001), os itens deste fator representam condutas apresentadas pelo indivíduo no sentido de aproximação do problema com a finalidade de manejar a situação estressora modificando-a. Este direcionamento do enfrentamento pode ocorrer de diferentes maneiras, tanto através da busca de ajuda para a resolução do problema ("ajuda prática de outras pessoas"), quanto pela reelaboração do significado da situação estressora ("reestruturação cognitiva"), o que ocorreu com os pais em ambos os grupos.

Analisando os dados de enfrentamento dos participantes entre os Grupos Restropectivo (GR) e Atual (GA), houve prevalência pouco maior do fator da EMEP "Focalizado no problema" para os pais do GR, que apresentou mediana maior $(4,33)$ do que os do GA $(3,85)$. Dessa forma, pode-se considerar que ao usar este tipo de estratégia para enfrentar o diagnóstico de malformação do filho, os pais investiam um pouco mais em um processo de reelaboração do significado da situação estressora (o diagnóstico) conferindo a ela um novo sentido. $O$ fato de que a mediana desse fator foi maior para o GR pode ajudar a confirmar a hipótese anterior de que o tempo transcorrido desde a notícia inicial do diagnóstico e as frequentes demandas como cuidador podem contribuir para a elaboração do impacto da malformação.

O fato do segundo tipo de enfrentamento mais observado ser a "Busca de prática religiosa/pensamento fantasioso" é, no mínimo, curioso, uma vez que não constatamos estudos que apontem para o fato de que seja comum homens adotarem a prática religiosa no enfrentamento de problemas. Pelo contrário, a estratégia "Busca de práticas religiosas/pensamento fantasioso" tem sido indicada por Seidl et. al (2001), em seu estudo de validação da EMEP para a população brasileira, como característica do gênero feminino, independente do estressor.

No entanto, quando analisado o enfretamento daqueles homens em comparação ao das mães e/ou companheiras, o mesmo tipo de enfrentamento, "Busca de prática religiosa/pensamento fantasioso", foi o mais prevalente também para as mulheres. Isto parece sugerir 
que o modo como os pais enfrentam a situação de malformação de um filho é semelhante ao da companheira, ou seja, focado na "Busca de prática religiosa". Também é relevante discutir o fato de que mais da metade deles $(66,6 \%)$ eram praticantes de uma religião, o que também pode ter influenciado neste resultado.

Cabe destacar que esse tipo de enfrentamento quando identificado pela EMEP relaciona-se tanto a itens relativos a estratégias focadas na "Busca de prática religiosa" quanto focadas no uso de "Pensamento fantasioso", o que deve ser discutido, uma vez que este último evidencia um caráter negativo a esse tipo de enfrentamento. No entanto, a literatura considera que o enfrentamento ou coping religioso deve ser considerado como uma possibilidade de manejo do estresse e das consequências negativas de eventuais eventos através do uso da religião, espiritualidade ou fé (Pargament, 1997). Nessa perspectiva, a religião, que representa uma estratégia de enfrentamento baseada em diferentes comportamentos, emoções, cognições e relações, pode assumir várias funções e, assim, contrariar a ideia estereotipada de que seria uma estratégia meramente defensiva, passiva, focada na emoção ou em formas de negação (Pargament, 1997; Panzini, 2009).

Assim, a estratégia de enfrentamento focada na busca por práticas religiosas pode funcionar tanto para ajudar na busca de um significado, quanto para controle ou conforto espiritual diante da situação (Pargament, 1997; Panzini, 2009), o que foi observado nos relatos dos participantes acerca das repercussões positivas do diagnóstico de malformação na relação do casal. De todo modo, não se pode esquecer que, segundo Koenig (2001), as crenças religiosas podem conferir tanto sentido positivo quanto negativo às experiências de vida, de acordo com a visão de mundo adotada pelo indivíduo, resultando, assim, em efeitos benéficos ou prejudiciais, a depender da interpretação pessoal da situação vivenciada.

Os tipos de enfrentamento que apareceram em menor proporção entre os pais foram os fatores "Busca de suporte social" em terceiro lugar; e o "Focalizado na emoção", que foi o menos prevalente em ambos os grupos de pais. A "Busca de suporte social" se caracteriza pela procura por apoio emocional ou informações como estratégias para enfrentar a situação estressora (Seidl et. al. 2001). Tal dado pode sustentar a ideia de que os homens apresentam mais dificuldade para falar de suas experiências e buscar ajuda, considerado como parte da própria tradição cultural brasileira que exige do pai ser sinônimo de força (Barros, Menandro, \& Trindade, 2006), sem fraquejar ou demonstrar sua sensibilidade. Isto também foi observado nos relatos coletados no Questionário "Momento da Notícia" quando $58,2 \%$ dos pais sequer sentiram tal necessidade ou se sentiram necessidade de falar com alguém sobre seu problema, não procuraram esse suporte. Por exemplo, um dos pais, Ananias, 
relatou que, apesar de ter sentido necessidade de conversar com alguém, não o fez, tendo conversado apenas consigo mesmo, reforçando a ideia que os homens apresentam, em geral, dificuldade em expressar verbalmente suas emoções.

O fator "Focalizado na emoção" foi o menos frequente em nossa amostra. Este tipo de enfrentamento se baseia em esforços com intenção de ignorar o estressor, usando até mesmo uma perspectiva fantasiosa que inibe reações comportamentais e emocionais, podendo inclusive acarretar mais problemas (Carver \& Connor-Smith, 2010). Apesar da notícia de malformação ser um tanto mobilizadora de recursos psíquicos e emocionais, este tipo de enfrentamento foi 0 menos utilizado e isso pode ser considerado positivo na medida em que não se trata de um enfrentamento resolutivo ou adequado para lidar com problemas, tal como o enfrentamento focalizado no problema.

Por fim, ainda que não tenham sido observados indicadores de depressão em níveis mais importantes (acima do mínimo) nos pais de ambos os grupos, tal dado deve ser analisado com reserva devido a limitação da amostra que não permite maior generalização dos dados. A literatura PSI, em geral, fala sobre a depressão paterna no contexto pós-natal (Edmondson et al., 2010; Fisher, Kopelman \& O'Hara, 2012; Massoudi, Hwang , \& Wickberg, 2013), não tendo sido encontrados estudos que abordassem a depressão paterna durante a gestação com diagnóstico de malformação. A depressão pós-natal paterna ou masculina pode estar associada com a depressão pósparto materna ou feminina (Iaconelli, 2011) e desfechos desfavoráveis no desenvolvimento infantil (Edmondson et al., 2010; Fisher, Kopelman \& O’Hara, 2012; Massoudi, Hwang \& Wickberg, 2013). Falceto et. al (2012) ainda acreditam que os sintomas depressivos no pai podem aparecer devido ao estresse que "o medo de falhar nas tarefas de provedor, apoiador emocional e parceiro romântico" (p. 294) podem causar no homem.

Limitações metodológicas do estudo não permitem corroborar ou refutar tais afirmativas, uma vez que os instrumentos usados não permitiram investigar as crenças dos pais acerca de sua função paterna. Para minimizar tais limitações, os dados de análise de conteúdo dos questionários foram extensamente analisados a fim de identificar semelhanças e diferenças entre os participantes dos dois grupos, levando-se em conta a diferença do tempo da notícia do diagnóstico de malformação, antes e depois do filho ter nascido. Apesar das tentativas de usar um enfoque multi metodológico para investigação do enfrentamento paterno, não foi possível identificar diferenças importantes nos dois grupos de pais, uma vez que a análise dos dados indicou uma vivência muito semelhante entre eles. I gualmente, o instrumento utilizado para avaliação da depressão não era especifico para avaliação de indicadores de depressão pós-natal, 
especialmente em pais. Estudos com a Escala de Depressão Pós-natal de Edimburgo para avaliar a depressão pós-natal paterna sugerem que este instrumento é sensível para rastrear este transtorno em pais após o parto (Edmondson et al., 2010; Fischer, Kolpman , \& O'Hara, 2012). Dessa forma, sugere-se a realização de futuros estudos adotando-se instrumentos específicos para depressão pós-natal, com amostra mais ampla que permita uma maior generalização dos resultados.

Conclui-se que os resultados deste estudo, ainda que preliminares, reforçam a importância de considerarmos as necessidades, sentimentos e particularidades do pai diante de um diagnóstico de malformação congênita de um filho. De acordo com o que se pôde observar, a notícia de malformação de um filho tem implicações e impacto emocional sobre o funcionamento psíquico do pai, já que resulta em sentimentos negativos os quais, com o decorrer do tempo, podem até ser elaborados e superados, especialmente diante de estratégias de enfrentamento mais resolutivas e adaptativas.

\section{Considerações finais}

Apesar da vida psíquica dos homens também sofrer impactos, a figura masculina foi construída social, histórica e culturalmente como o "sexo forte" e por isso existe grande resistência em aceitar a ideia de que homens se sentem abalados por determinadas notícias e acontecimentos, o que seria sinal de possível fraqueza. Essa dificuldade pode partir tanto do próprio homem, que julga ter que ser forte para dar apoio para a parte mais fraca (a mulher), como da sociedade, que exige que ele seja forte e inabalável diante de situações de desafio.

Assim, é importante ressaltar a adoção de medidas de prevenção aos riscos à saúde mental do pai, nessa condição de vulnerabilidade. Nesta perspectiva, o profissional de psicologia pode ser um mediador e facilitador da expressão dos sentimentos e pensamentos por parte deste homem, que também se encontra em processo de reelaboração de suas expectativas e sonhos pelo filho saudável e idealizado perdido e a constatação de um filho real diagnosticado com malformação.

Por último, faz-se necessário alertar os profissionais de saúde para a importância de prestar assistência e oferecer atenção não apenas à gestante, mas, também, ao pai do bebê ainda durante a gestação, a fim de auxiliar no enfrentamento do impacto emocional que um diagnóstico de malformação congênita pode gerar na tríade pai-mãefilho e sua família. 


\section{Referências}

Barros, S. M. M., Menandro, P. R. M., \& Trindade, Z. A. (2006) Vivências paternas em UTI neonatal. Psicologia Hospitalar, 4(2), 1-18. Recuperado em 06 de agosto, 2014, de http: // pepsic. bvsalud.org/scielo.php?script=sci_arttext\&pid=S1 677-74092006000200003\&lng=pt\&tIng $=p t$

Benute, G. R. G., Nomura, R. N. Y, Liao, A. W., Brizot, M. L. Lucia, M. C. S., \& Zugaib, M. (2012). Feelings of women regarding endof-life decision making after ultrasound diagnosis of a lethal fetal malformation. Midwifery, 28, 472-475.

Bornholdt, E. A, Wagner, A., \& Staudt, A. C. P. (2007) A vivência da gravidez do primeiro filho à luz da perspectiva paterna. Psicologia Clínica 19, 75-92. Recuperado em 28 de Outubro, 2014, de http://www.scielo.br/pdf/pc/v19n1/06.pdf

Bossardi, C. N., \& Vieira, M. L. (2010). Cuidado paterno e desenvolvimento infantil. Revista de Ciências Humanas, 44(1), 205-221.

Carver, C. S., \& Connor-Smith, J. (2010). Personality and Coping. Annual Review of Psychology, 61, 679-704.

Cunha, J. A. (2001). Manual da versão em português das escalas Beck. São. Paulo: Casa do Psicólogo.

Cia, F., Williams, L. C. A., \& Aiello, A. L. R. (2005) Influências paternas no desenvolvimento infantil: revisão de literatura. Relacionamento pai-filho. Psicologia Escolar e Educacional, 9(2), 225-233. Recuperado em 12 de junho, 2014, de http://www.scielo.br/pdf/pee/v9n2/v9n2a05.pdf

Edmondson, O. J. H., Psychogiou, L., Vlachos, H., Netsi, E., \& Ramchandani, P. G. (2010). Depression in fathers in the postnatal period: Assessment of the Edinburgh Postnatal Depression Scale as a screening measure. Journal of Affective Disorders, 125, 365-368.

Falceto, O. G., Fernandes, C. L., \& Kerber, S. R. (2012). Alerta sobre a depressão pós-parto paterna. Revista Brasileira de Ginecologia e Obstetrícia, 34(7), 293-295. Recuperado em 12 de novembro, 2014, de http://www.scielo.br/pdf/rbgo/v34n7/01.pdf

Field, T. (2010). Postpartum Depression Effects on Early Interactions, Parenting, and Safety Practices: A Review. Infant Behavior Development Journal, 33(1), 1. doi: 10.1016/j.infbeh.2009.10.005

Fisher, S. D., Kopelman, R., \& O'Hara, M. W. (2012). Partner report of paternal depression using the Edinburgh Postnatal Depression Scale-Partner. Arch Womens Ment Health, 15, 283288. 
Gomes, A. G., \& Piccinini, C. A. (2010) Malformação no bebê e maternidade: aspectos teóricos e clínicos. Psicologia Clínica, 22(1), 15-38. Recuperado em 09 de julho, 2014, de http://www.scielo.br/pdf/pc/v22n1/a02v22n1

Ha, J. F., Anat, D. S., \& Longnecker, N. (2010). Doctor-Patient Communication: A Review. The OchsnerJ ournal, 10(1), 38-43. Recuperado em 29 de setembro , 2014, de http: // www. ochsnerjournal.org/doi/pdf/10.1043/TOJ-09-0040.1 Iaconelli, V. (2011) Depressão pós-parto masculina. Instituto Brasileiro de Psicologia Perinatal Gerar. Recuperado em 08 de agosto, 2014, de http://www.institutogerar.com.br/artigos/23_ARTIGO_DEPRESS \% C3\% 830\% 20POS\% 20PARTO\% 20MASCULINA. pdf

J ungmarker, E. B., Lindgren, H., \& Hildingsson, I. (2010). Playing second fiddle is okay - Swedish fathers' experiences of prenatal care. J Midwifery Womens Health 55(5), 421-9. Recuperado em 30 de Setembro, 2014, de http://onlinelibrary. wiley.com/doi/10.1016/j.jmwh.2010.03.007 /abstract

Koenig, H. G. (2001). Religion and Medicine III: developing a theoretical model. Journal Psychiatry Med, 31 (2), 199-216.

Lazarus, R. S., \& Folkman, S. (1984). Stress, appraisal, and coping. New York: Springer.

Locock, L., \& Alexander, J. (2006) 'Just a bystander'? Men's place in the process of fetal screening and diagnosis. Social science \& medicine, 62(6), 1349-1359. Recuperado em 28 de Agosto, 2014, de http://www.ncbi.nlm.nih.gov/pubmed/16165260

Machado, M. E. C. (2012). Casais que recebem um diagnóstico de malformação fetal no pré-natal: uma reflexão sobre a atuação do Psicólogo Hospitalar. Revista da Sociedade Brasileira de Psicologia Hospitalar, 15(2), 86-95. Recuperado em 02 de abril, 2014, de http://pepsic. bvsalud.org/scielo. php?pid=S1516$08582012000200007 \&$ script $=$ sci arttext

Massoudi, P. Hwang, P., \& Wickberg, B. (2013). How well does the Edinburgh Postnatal Depression Scale identify depression and anxiety in fathers? A validation study in a population based Swedish sample. Journal of Affective Disorders, 149, 67-74.

Montenegro, C. A. B., \& Rezende, J., Filho. (2013) Obstetrícia. Rio de Janeiro: Guanabara Koogan.

Panzini, R. G. (2004). Escala de Coping Religioso-espiritual (Escala CRE): tradução, adaptação e validação da Escala RCOPE, abordando relações de saúde e qualidade de vida. Dissertação de Mestrado. Universidade Federal do Rio Grande do Sul. Rio Grande do Sul, RS, Brasil.

Pargament, K. I. (1997). The psychology of religion and coping: Theory, research, practice. New York: Guilford Press. 
Perosa, G. B., Canavez, I. C., Silveira, F. C. P., Padovani, F. H. P., \& Peraçoli, J. C. (2009) Sintomas depressivos e ansiosos em mães de recém-nascidos com e sem malformações. Revista Brasileira de Ginecologia \& Obstetrícia, 31(9), 433-439. Recuperado em 19 de agosto, 2014 em http: //www. producao.usp. br/bitstream/handle/BDPI/7243/art CANAVEZ_Sintomas_depressivos_e_ansiosos_em_maes_de_20 09. pdf? sequence $=1$

Piccinini, C. A., Silva, M. R., Gonçalves, T. R., Lopes, R. S., \& Tudge, J. (2004) O envolvimento paterno durante a gestação. Psicologia: Reflexão e Crítica, 17(3), 303-314. Recuperado em 18 de agosto, 2014, de http://www.scielo.br/scielo.php?script=sci_arttext\&pid=S010279722004000300003\&lng=pt\&tlng=pt. doi: 10.1590/S010279722004000300003

Ramos, T. C. L., Hoffmann, V. M. B., \& Regen, M. (1985). As dificuldades em transmitir a notícia: pesquisa junto a pais de pacientes portadores de Síndrome de Down. Revista Brasileira de Deficiência Mental, 18, 47-69.

Roecker, S., Mai, L. D., Baggio, Simone C., Mazzola, J. C., \& Marcon, S. S. (2012). A vivência de mães de bebês com malformação. Escola Anna Nery, 16(1), 17-26. Recuperado em 29 de Setembro, 2014 ,

de http: //www.scielo.br/scielo.php?script=sci_arttext\&pid=S1414$81452012000100003 \&$ Ing=en\&tIng=pt. doi: 10.1590/S141481452012000100003

Seidl, E. M. F., Tróccoli, B. T., \& Zannon, C. M. L. C. (2001) Análise Fatorial de Uma Medida de Estratégias de Enfrentamento. Psicologia: Teoria e Pesquisa, 17(3), 225-234. Recuperado em 17 de agosto, 2014, de http: //www.scielo.br/scielo.php?script=sci_arttext\&pid=S0102$37722001000300004 \&$ Ing =en\&tlng=pt. doi: 10.1590/S010237722001000300004

Vasconcelos, L., \& Petean, E. B. L. (2009) O impacto da malformação fetal: indicadores afetivos e estratégias de enfrentamento das gestantes. Psicologia, Saúde e Doenças, 10(1), 69-82. Recuperado em 25 de julho, 2014, de http: //www. redalyc. org/articulo. oa? id $=36219059006$

Vieira, M. L., Bossardi, C. N., Gomes, L. B., Bolze, S. D. A., Crepaldi, M. A., \& Piccinini, C. A. (2014). Paternidade no Brasil: revisão sistemática de artigos empíricos. Arquivos Brasileiros de Psicologia, 66(2), 36-52.

Endereço para correspondência Elisabeth Hellen Pereira da Silva Universidade Federal do Rio de Janeiro 
Instituto de Psicologia - Campus Praia Vermelha

Avenida Pasteur, 250, fds, CEP 22021-001, Rio de Janeiro - RJ, Brasil

Endereço eletrônico: elisabethhellen@gmail.com

\section{Emmanuela Rocha da Cruz Girão}

Universidade Federal do Rio de J aneiro

Instituto de Psicologia - Campus Praia Vermelha

Avenida Pasteur, 250, fds, CEP 22021-001, Rio de Janeiro - RJ, Brasil

Endereço eletrônico: emmanuelagirao@gmail.com

\section{Ana Cristina Barros da Cunha}

Universidade Federal do Rio de J aneiro

Instituto de Psicologia - Campus Praia Vermelha

Avenida Pasteur, 250, fds, CEP 22021-001, Rio de Janeiro - RJ, Brasil

Endereço eletrônico: acbcunha@yahoo.com.br

Recebido em: 23/11/2014

Reformulado em: 25/09/2015

Aceito para publicação em: 09/11/2015

\section{Notas}

* Graduanda em Psicologia no Instituto de Psicologia e estagiária na MaternidadeEscola, Universidade Federal do Rio de Janeiro, Rio de Janeiro / RJ, Brasil.

** Graduanda em Psicologia no Instituto de Psicologia e estagiária na MaternidadeEscola, Universidade Federal do Rio de Janeiro, Rio de Janeiro / RJ, Brasil.

*** Professora associada do Instituto de Psicologia e Coordenadora acadêmica da área de Psicologia do Programa de Residência Multiprofissional Integrada em Saúde Perinatal da Maternidade-Escola, Universidade Federal do Rio de Janeiro; Professor colaborador do Programa de Pós-graduação em Psicologia, Universidade Federal do Espírito Santo.

1 A malformação, de acordo com Montenegro e Rezende Filho (2013), é uma anomalia fenotípica resultante de uma alteração precoce da embriogênese que leva a um defeito morfológico de um órgão, parte dele ou área do corpo.

${ }^{2}$ Considera-se coping como uma constante mudança cognitiva e comportamental utilizada para manejar demandas externas e/ou interna específicas, que são avaliadas como sobrecarregando ou excedendo os recursos da pessoa (Lazarus \& Folkman, 1984, p.141 e 142).

3 Todos os nomes usados são fictícios a fim de manter o sigilo e anonimato dos participantes. 